\title{
Geometric Abundance Estimation Using Variable Endmembers for Hyperspectral Imagery
}

\author{
Huadong Yang and Jubai An \\ College of Information Science and Technology \\ Dalian Maritime University, Dalian 116026, China \\ yanghd8608@dlmu.edu.cn, jubaian@sohu.com
}

\begin{abstract}
Abundance estimation is an important step of quantitative analysis of hyperspectrat remote sensing data. Due to physical interpretation, sum-to-one and non-negativity constraints are generally imposed on the abundances of materials. This paper presents a geometnc approach to fully constrained linear spectral unmixing using variable endmember sets for the pixels. First, an improved method for selecting per-pixel candidate endmember set is presented, which is suitable for dealing with hyperspectral image with large number of endmembers. To determine the optimal per-pixel endmember set from the entire endmembers present in the hyperspectral scene, an iterative partially constrained geometrc unmixing is then performed, in which subspace projection is used for full y constrained least square estimation. The performance of the resulting unmixing algokithm is evalated by comparison with benchmark unmixing algorithm on synthetic and reahyperspectral data.
\end{abstract}

Keywords: Abundance Estimation, Full Constraint, Subspace Projection, Hyperspectral Image

\section{Introduction}

Hyperspectral remote sensing jmagety has been widely used in various applications as its rich spectral information provides an effective way of distinguishing different objects. However, due to the low spatial resolution of the sensors, the pixels may contain spectra from several distinct material substances and be viewed as the "mixed pixels". To utilize the hyperspectral data, these mixed pixels must be decomposed into a set of pure class spectra, called endmembers anctheir corresponding fractions, called fractional abundances or simply abundances. Hyperspectral unmixing is a technique for this purpose. The process of hyperspectral unmixing usually involves two steps, extracting the endmembers and estimating the abundance of each endmember for each pixel in the image [1]. Most unmixing algorithms require several additional steps, such as denoising of the data, dimensionality reduction (DR), or estination of the number of the endmembers, and some unmixing algorithms integrate all the steps simultaneously (See [2] for a recent and complete overview of hyperspectral unmixing). In this paper, we focus on the abundance estimation step of the hyperspectral unmixing process.

Although nonlinear mixing model has attracted increasing attention in recent years, linear mixing model (LMM) is still widely used in the past decade to address spectral unmixing problem. The reason is twofold [3]: 1) in spite of its simplicity, LMM is an acceptable approximation for the light scattering in many real scenarios; 2) under the LMM, spectral unmixing is interpretable as a blind source separation problem or a nonnegative matrix factorization problem, which have been vastly researched in many signal processing areas. 
In LMM, the observed spectral signature in each pixel can be expressed as a linear combination of the endmember signatures, weighted by their abundances. Since the number of spectral bands usually exceeds the number of endmembers in the image, the spectral unmixing problem is cast in terms of an over-determined system of equations. For endmember fractions to be meaningful, abundances must sum to one (ASC) and be nonnegative (ANC) [4]. The fully constrained abundance estimation approach should implement these two constraints simultaneously. The researchers have noted that the former constraint is easy to implement [5], for instance, by using lagrangian multipliers, the latter one, however, is difficult to deal with, since it results in a set of inequalities and does not have a closed-form solution. To this end, many approaches which require more advanced optimization methods have been proposed, including nonnegative matrix factorization technique [6][7], blind signal separation technique [8], as well as general iterative techniques [5]. However, solutions to these problems involve computational extensive algorithms, which are not appropriate for a large number of pixels.

To overcome the heavy computational demands, simplex volume based abundance estimation approaches have been proposed, which exploit the fact that the mixed pixels lie inside the simplex, endmembers correspond to the vertices of simplex, and thus the fractional abundances can be expressed as the baryeentric coordinates asing a geometric formulation [9]. Geng et al. [10] and Honeine et al. [11] have proposed positive volume based and signed volume based unmixing methods in original and reduced space, respectively. However, solutions to them cannot obey the full constraint simultaneously.

The new approach proposed in this paper is named geometric estimation based on subspace projection using variable endmembers (GESPVE). This method exploits the common-sense fact that the number of endmembers that participates in the composition of a specific pixel is less than the one present in entire hyperspectral image, especially for which covering a large geospatial area. With this in mind, we incorporated the subspace projection technique into the process of geometric abundance estimation, and adopted an iterative search trategy to minimum residual, rather than an exhaustive one used in [12], which leads to extremely expensive computing time. The performance of the proposed unmixing algorithm is evaluated by comparison with well-known fully constrained least square spectral ynmixing (FCLSU) [5] algorithm on simulation and real hyperspectral data.

\section{Linear Mixing Model and Geometric Abundance Estimation}

\subsection{Linear Mixing Model}

Most of hyperspectral unmixing algorithms adopt LMM in which a mixed pixel is a linear combination of endmembers and the linear coefficient of each endmember is its abundance The hyperspectral unmixing based on LMM is referred to as linear spectral unmixing (LSU) or as linear spectral mixture analysis (LSMA). Specifically, LSMA model a data sample vector $x=\left(x_{1}, x_{2}, \cdots, x_{L}\right)^{T}$ as a linear mixture described by

$$
\boldsymbol{x}=\sum_{i=1}^{p} \alpha_{i} \boldsymbol{e}_{i}+\boldsymbol{n}
$$

where $e_{i}=\left[e_{i, 1}, e_{i, 2}, \cdots, e_{i, L}\right]^{T}$ denotes the $i$ th endmember, $\alpha_{i}$ is the abundance fraction of the $i$ th endmember in the pixel, $p$ is the number of endmembers present in the observed scene, $L$ is the number of available spectral bands for the image, and $\boldsymbol{n}$ takes into account possible errors. 
For endmember fractions to be physically meaningful, two constraints are generally imposed on (1), which are ASC and ANC specified by $\sum_{i=1}^{p} \alpha_{i}=1$, and $\alpha_{i} \geqslant 0$, for $1 \leqslant i \leqslant$ $p$, respectively. The matrix form of LMM is given by $\boldsymbol{x}=\mathbf{E} \boldsymbol{\alpha}+\mathbf{n}$, where $\mathbf{E}=\left[\begin{array}{llll}\boldsymbol{e}_{1} & \boldsymbol{e}_{2} & \cdots & \boldsymbol{e}_{p}\end{array}\right]$ is endmember signatures, $\alpha=\left[\begin{array}{llll}\alpha_{1} & \alpha_{2} & \cdots & \alpha_{p}\end{array}\right]^{\mathrm{T}}$ is a column vector of fractional abundance. According to convex geometry theory, if both ASC and ANC are satisfied, all the mixed or pure pixels will lie in a simplex spanned by all endmembers.

\subsection{Geometric Abundance Estimation}

This paper extends the traditional geometric abundance estimation approaches, and hence, we first simply introduce two representative method proposed in [10] and [11]. For convenient description, we call the former one PVAE, and the latter one SVAE.

In [10], the volume of the simplex spanned by $p$ endmembers $\left\{\boldsymbol{e}_{1}, \boldsymbol{e}_{2}, \ldots, \boldsymbol{e}_{p}\right\}$ and original point $\mathbf{O}$ is given by

Then, the abundance $\alpha_{i}$ is defined as

$$
V\left(\mathbf{O}, \boldsymbol{e}_{1}, \boldsymbol{e}_{2}, \cdots, \boldsymbol{e}_{p}\right)=\frac{1}{(p-1) !} \sqrt{\operatorname{det}(\mathbf{E} \mathbf{E})}
$$

$$
\alpha_{i}=\frac{V_{S \backslash\left\{e_{i}\right\} \cup\{x\}}}{V_{S}}=\frac{V\left(\mathbf{O}, e_{1}, \ldots, e_{i},-\boldsymbol{x}, e_{i+1}, \ldots \boldsymbol{e}_{p}\right)}{V\left(\mathbf{O}\left(e_{1}\right) e_{2}, \ldots, e_{p}\right)}, i=1,2, \ldots, p
$$

where $\backslash$ denote the set difference operator, and $S \backslash\left\{e_{i}\right\} \cup\{x\}$ is the set defined by $S$, where $\boldsymbol{e}_{i}$ has been removed and $\boldsymbol{x}$ added.

Using above expression to obtain fractionalabundances is straightforward and free of DR. However, it does not guarantee that the estimated fractional abundances satisfy the ASC. Similarly, in [11] the abundances are expressed as the signed volume of the simplex in reduced space rather than in original spectral space. The benefit of this is that the abundances al ways obery the ASC.

\section{Table 1 Comparison of Two Geometric Abundance Estimation Methods}

\begin{tabular}{|c|c|c|c|}
\hline Method & DR & Violation of ASC & Violation of ANC \\
\hline PVAE & No & No & Yes \\
\hline SVAE & Yes & Yes & No \\
\hline
\end{tabular}

Table 1 shows the comparison results of PVAE and SVAE. As shown by the table, both PVAE and SVAE cannot meet the ASC and ANC constraints simultaneously. To this end, we will extend the PVAE to satisfy the ASC constraint in the following.

\section{Methodology}

According to the position relationship between the mixed pixel and simplex, we divide the mixed pixels into two types as follows: the interior pixels, i.e., the pixels inside the simplex, and the exterior pixels, i.e., the pixels outside the simplex. As stated above, the ASC and ANC constraints constrain the mixed pixel to be in the simplex spanned by the endmember vertices. That is to say that the abundance will violate the full constraint when a mixed pixel is the exterior one. In this case, solving the fully 
constrained unmixing problem can be turned into searching for the projection of this exterior pixel onto the simplex spanned by an optimal endmember set, and then using (2) to obtain the abundances by replacing the exterior pixel with its projection. With this in mind, we present a fully constrained spectral unmixng algorithm in the following subsection.

\subsection{Selection of Candidate Endmembers Set}

The image scene captured by the hyperspectral sensor usually cover a large geospatial area, and thus the number of endmembers is quite large but only a small subset of these endmembers may actually participate in the composition of a specific pixel. For this reason, we present a method by modifying an available approach developed in [13], which can assist in the selection of optimal per-pixel endmember set.

The improved method uses a recursive process based on the Gramm-Schmidt orthogonallization to select the candidate endmembers

$$
\boldsymbol{x}_{r}=\boldsymbol{x}-\omega \cdot c_{\max } \cdot \hat{\boldsymbol{e}}_{\max }
$$

where $\boldsymbol{x}_{r}$ is a residual vector, $\omega$ is a parameter and $0<\omega \leqslant 1, \hat{\boldsymbol{e}}_{\max }$ is normal endmember vector with maximum projection.

First, each pixel is projected onto all norman ndmember vectors, and the most efficient projection, which corresponds to that with the highest value $c_{\max }$, indicates the first selected endmember. Then, the residual $x_{n}$ is repeatedly projected onto each of the remaining endmembers to find the second selected endmember.

This process continues continue up to the identification of a complete subset with a prefixed maximum number of endmembers, or until no positive residual remains. After this process, the candidate endmember set is oblained for each pixel.

\subsection{Sub-Simplex Plane Projection}

The main goal of this study is to find an appropriate set of endmembers for each pixel so that the projection of the exterior pixel on the sub-simplex plane through these endmmebers could lie in the simplex. So, we first address the projection problem. Note that this subsimplex plane in fact is the hyperplane through these endmember vertices. As a result, the projection on the sub-simplex plane,is turned into the projection onto the hyperplane.

According to knowledge of linear algebra, the projection of point $\boldsymbol{x}$ on hyperplane through the endmember vertices $\left\{\boldsymbol{e}_{1}, \boldsymbol{e}_{2}, \ldots, \boldsymbol{e}_{p}\right\}$ can be obtained by adapting the projection onto the subspace, i.e., following expression

$$
\boldsymbol{x}^{\prime}=\mathbf{E}\left(\mathbf{E}^{\mathrm{T}} \mathbf{E}\right)^{-1} \mathbf{E}^{\mathrm{T}}\left(\boldsymbol{x}-\boldsymbol{e}_{1}\right)+\boldsymbol{e}_{1}
$$

It should be noted that $\boldsymbol{e}_{1}$ can be replaced by any one in $\left\{\boldsymbol{e}_{2}, \boldsymbol{e}_{3}, \ldots, \boldsymbol{e}_{p}\right\}$.

\subsection{GESPVE Algorithm}

The candidate endmember set (denoted by $\mathbf{C}$ ) is often not the true optimal one (denoted by $\mathbf{O})$, so we consider two typical relationships between $\mathbf{C}$ and $\mathbf{O}$, i.e., $\mathbf{C} \subset \mathbf{O}$ and $\mathbf{C} \supset \mathbf{O}$, and present two iterative algorithms to search optimal endmember set for pixel $\boldsymbol{x}$ and $\mathbf{E}$ as follows: 
Algorithm 1: $(\mathbf{C} \subset \mathbf{O})$

Step 1. set $i=1$, construct the difference set $\mathbf{R}=\mathbf{E}-\mathbf{C}$.

Step 2. construct $\mathbf{B}=\mathbf{C} \cup \boldsymbol{r}_{i}$, where $\boldsymbol{r}_{i} \in \mathbf{R}$.

Step 3. calculate the projection of $\boldsymbol{x}$ on the simplex plane formed by $\mathbf{B}$, denoted by $\boldsymbol{x}_{\mathbf{B}}^{\phi}$, obtain the abundance of $\boldsymbol{x}_{\mathrm{B}}^{\mathrm{c}}$ by (2) using $\mathbf{E}$.

Step 4. if the abundance are fully constrained, then set $\mathbf{C}=\mathbf{B}$, and go to Step 1, otherwise update $i=$ $i+1$.

Step 5. if $\boldsymbol{r}_{i} \in \mathbf{R}$, go to Step 2, else output the optimal per-pixel endmember set $\mathbf{C}$ and the corresponding abundance

Algorithm 2: $(\mathbf{C} \supset \mathbf{O})$

Step 1. set $i=1$, construct $\mathbf{B}=\mathbf{C}-\boldsymbol{c}_{i}$, where $\boldsymbol{c}_{i} \in \mathbf{C}$.

Step 2. calculate the projection of $\boldsymbol{x}$ on the simplex plane formed by $\mathbf{B}$, denoted by $\boldsymbol{x}_{\mathrm{A}}^{\prime}$, obtain the abundance of $x_{\mathrm{B}}^{\prime}$ by (2) using $\mathbf{E}$

Step 3. if the abundance are not fully constrained, then set $\mathbf{C}=\mathbf{B}$, go to Step 1, else output the optimal per-pixel endmember set $\mathbf{C}$ and the corresponding abundance

Based on the above statement, the process of searching for the fulty constrained abundance is described as follows:

GESPVE algorithm:

Step 1. select candidate endmember set $\mathbf{C}$ for the p xel $\boldsymbol{x}$ using (3), calculate $\mathbf{R}=\mathbf{E}-\mathbf{C}$

Step 2. Calculate $x_{\mathrm{C}}^{\prime}$, obtain its abundanee by (2) using

Step 3. if the abundances are fully constrained and $y$, output the abundances as the optimal fully constrained solution to (1). If the abundances are fully constrained but $x_{\mathrm{C}}^{\prime} \neq \boldsymbol{x}$, go to Step 4; otherwise go to Step 5 .

Step 4. perform algorithm 1, and output the results.

Step 5. perform algorithm 2 use its outputs as inputs to perform algorithm 1 .

\section{Experimental Results and Ánalysis}

In this section, we conduct a series of experiments on synthetic and real hyperspectal data to demonstrate the effectiveness of the proposed algorithm. Two algorithms: FCLSU and PVAE were considerea lor comparison purposes.

To quantitatively evaluate the performances of these algorithms, root mean square error (RMSE) is used as the criteria for abundance estimation. For synthetic image, the RMSE is defined as follow

$$
\mathrm{RM} \mathrm{SE}_{k}=\sqrt{\frac{1}{N} \sum_{j=1}^{N}\left(\alpha_{k, j}-\hat{\alpha}_{k, j}\right)^{2}}
$$

where $N$ is the number of data points (pixels), $\alpha_{k, j}$ and $\hat{\alpha}_{k, j}$ are the resulting and the ground-truth abundance for the $k$ th endmember in the $j$ th pixel, respectively. The average value of all RMSE is used to evaluate the performance of the algorithms.

Table 2. Specifications of the PC and Software Used for Experiments

\begin{tabular}{|c|c|c|c|}
\hline CPU & Memory & OS & Software \\
\hline Pentium (R) 4 & \multirow{2}{*}{2 GBytes } & Windows XP Professional & Matlab \\
CPU 3.2GHz & & with Service Pack 3 & R2009b \\
\hline
\end{tabular}


To compare the computing time, the same computing environment of the specifications of the PC and software used to perform all the versions of GESPVE is specified in Table 2.

\subsection{Experiments on Synthetic Data}

In all experiments, the synthetic data are linearly mixed with the known endmembers and abundances according to LMM. The endmembers signatures are selected from the USGS spectral library [14], and the abundances were generated according to Dirichlet distribution [15] whose density enforces the sum-to-one and non-negativity constraints. The noise was added using the following expression

$$
\boldsymbol{n}=M \cdot\left(\frac{q(0,1)}{\mathrm{SNR}}\right) \cdot
$$

where $q$ is a randomly generated number with a mean of zero and a standarcdeviation of one, SNR is signal-to-noise ratio, and $M$ is the assumed reflectance for the spectrum. According to Harsanyi [16], the SNR is defined as half the reflectance divided by the standard deviation of the noise, i.e., $M=0.5$.

The algorithms are evaluated by five experiments. The first experiment aims to test the numerical accuracy by comparing the abundance. Experiments 2.5 aim to test the computational performance by calculating the computing time for several parameter sets with GESPVE, FCLSU, and PVAE algorithms and comparing them one by one.

Table 3. Comparison of Three Algorithms with Different Noise Level

\begin{tabular}{|c|c|c|c|c|c|c|}
\hline \multirow{2}{*}{ Algorithm } & \multicolumn{7}{|c|}{ SNR } \\
\cline { 2 - 7 } & $15 \mathrm{~dB}$ & $30 \mathrm{~dB}$ & $50 \mathrm{~dB}$ & $70 \mathrm{~dB}$ & $90 \mathrm{~dB}$ & $110 \mathrm{~dB}$ \\
\hline PVAE & 0.1341 & 0.1058 & 0.0795 & 0.0623 & 0.0548 & 0.0504 \\
\hline FCLSU & 0.1036 & 0.0793 & 0.0614 & 0.0507 & 0.0449 & 0.0402 \\
\hline GESPVE & 0.0924 & 0.0715 & 0.0563 & 0.0485 & 0.0438 & 0.0399 \\
\hline
\end{tabular}

Experiment 1: In the first experiment, we tested the accuracy by comparing the estimated abundance with the rear one. Table 3 shows the results for $p=10, L=224, N$ $=5000$, and SNR varied from 15.1 to 110:1. As we can see from the table, the proposed algorithm outperforms the vell-known FCLSU algorithm, especially for high noise level case. The PVAE algorithm performs worst among of them. Specifically, for very high SNR, GESPVE slightly outperforms both FCLSU and PVAE. As the noise level increases, the performance of PVAE algorithm drops significantly. This shows that the fully constrained approaches outperform the partially constrained ones in generally. However, the GESPVE still performs better than the FCLSU.

Experiment 2: In this experiment, we limited the number of endmembers to six in each synthetic pixel, i.e., there were at most six endmember signatures per pixel. The same limit was adopted in the following experiments. Figure 1 (a) shows the computing time of the GESPVE, PVAE, and FCLSU algorithms with $L=224$, SNR $=15, N=5000$, as the number of endmembers varied from 3 to 15 . As shown by the figure, the computing time increases exponentially as a function of $p$ for the GESPVE, PVAE and FCLSU algorithms approximately. The performance of PVAE was best in terms of computing time. We notice that the GESPVE algorithm consumed more time than the FCLSU algorithm when the number of endmembers is relatively small, e.g., $p<8$ in this experiment. This is mainly due to the fact that the candidate endmembers selection method presented in Section 3.1 was not effective enough for GESPVE algorithm but spent relatively much time. 
Experiment 3: In this experiment, the impact of the number of data points on the GESPVE, PVAE and FCLSU algorithm's performance is studied. Figure 1 (b) shows the results of three algorithm with $p=10$, SNR $=15, L=224$, as the number of data points varied from 1000 to 50000. It is clear that the computing time of all algorithms scales linearly with the number of data points. However, the computing time of the FCLSU algorithm increase significantly for increasing $N$, and the proposed GESPVE algorithm outperform the FCLSU algorithm once again.
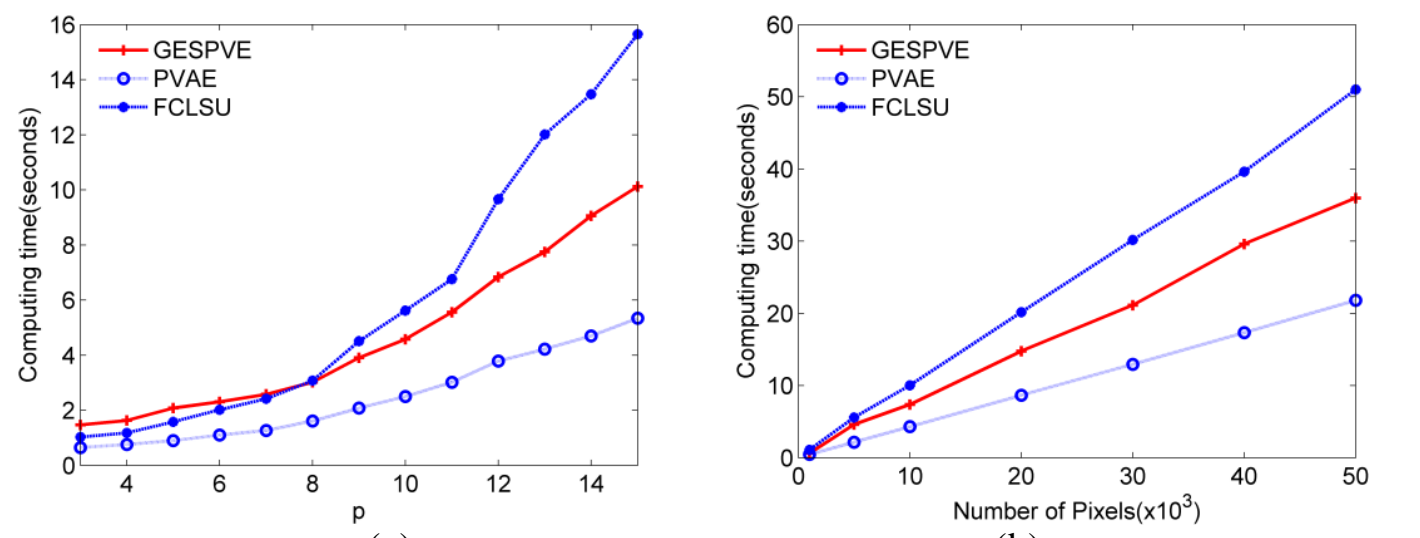

(a)
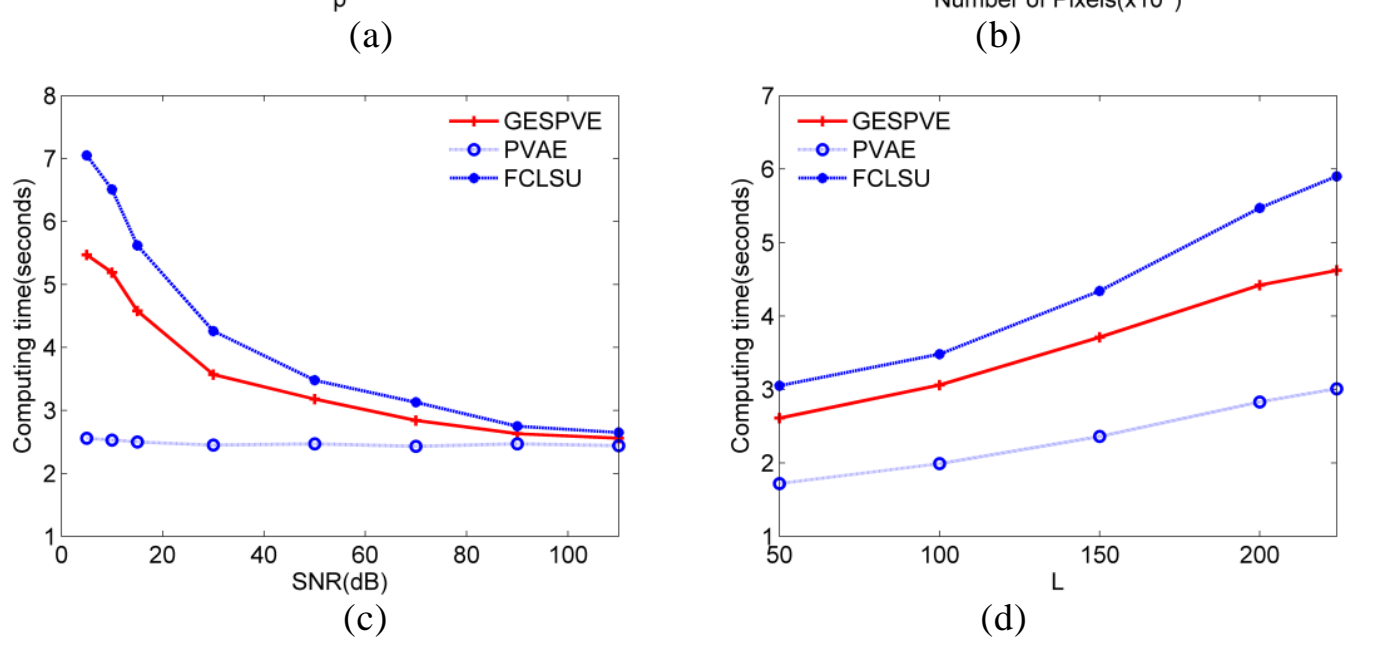

(d)

Figure 1. Computing Time of GESPVE, PVAE, and FCLSU Algorithms with (a) Number of Endmembers (b) Number of Pixels (c) Different Noise Level (d) Number of Dimensionality

Experiment 4: In this experiment, a comparative analysis on the issue of algorithm senstivity to noise is studied by simulating synthetic data with different noise levels. The SNR is varied to be $5 \mathrm{~dB}, 10 \mathrm{~dB}, 15 \mathrm{~dB}, 30 \mathrm{~dB}, 50 \mathrm{~dB}, 70 \mathrm{~dB}, 90 \mathrm{~dB}$ and $110 \mathrm{~dB}$. Figure 1 (c) shows the results with $p=10, L=224$, and $N=5000$. As we can see from the figure, the PVAE performs best, followed by the GESPVE whose performance is second and the FCLSU performs worst. Specifically, both GESPVE and FCLSU algorithms perform worse in terms of computing time as SNR decreases, while the result of PVAE is not related to the noise level. This is because the PVAE does not deal with the case where abundances do not satisfy the ASC constraint, but both GESPVE and FCLSU would do. 
Experiment 5: Finally, the dependence of computing time on the dimensionality is shown in Figure 1 (d), where $\mathrm{SNR}=15, p=10$, and $N=5000$. Experimental results show that the computing time of GESPVE, PVAE, and FCLSU algorithms increases as the dimensionality increases. This is because the computing time of three algorithms is mainly influenced either by the matrix inversion operation or by determinant of matrix, where the size of each matrix depends on the dimensionality. Once again, the performance of GESPVE algorithm was better than the one of FCLSU algorithm.

It should be noted that the value of parameter $\omega$ was not related to the numerical accuracy although it would affect the computational performance of the proposed GESPVE algorithm. According to experimental results, the parameter is set as $\omega=0.5$ in this paper.

\subsection{Experiments on Real Data}

In this subsection, we apply the GESPVE, PVAE, and FCLSU algorithms to the real hyperspectral data captured by the Airborne Visible/Infrared Imaging Spectrometer (AVIRIS) over Cuprite, Nevada. This data set has been widely used to validate the performance of the spectral unmixing algorithms [6], 1N, [17]. A sub-scene is used in the experiment, which consists of 200 lines and 200 pixels per line. To improve the unmixing performance, lower SNR and water-vapo absorption bands were removed from the original 224-band data cube. As a pesult, a totahof 188 reflectance channels are used in the experiment.

The estimated number of endmembers using the Hysime method [15] is equal to 18 and using the VD method [18] is equalco 10 . We applied the vertex component analysis (VCA) [17] algorithm to extract 18 endmembers of which some endmembers was extracted multiple times (e.g., Kablinite \#1 \#2, \#3; and Alunite \#1, \#2). It is worth mentioning that variants of the same mineral with slightly different spectra can be considered as the same endmember, and hence, we set $p=10$. Using these endmember signatures, GESPVE, FCLSU, and PVAE algorithms are used to estimate the abundances. The RMSE 1s, used for êvaluating unmixing performance

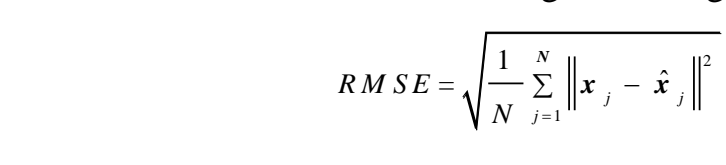

where $\boldsymbol{x}_{j}$ and $\hat{\boldsymbol{x}}_{j}$ are the th pixel vector and its reconstruction vector, respectively

Table 4 tabulates be computing time and RMSE for the GESPVE, FCLSU, and PVAE algorithms.

Table 4 Computing time of the Algorithms on AVIRIS Cuprite Data Set
\begin{tabular}{|c|c|c|c|}
\hline Algorithm & FCLSU & PVAE & GESPVE \\
\hline Computing time (second) & 102.75 & 35.43 & 86.16 \\
\hline RMSE & 0.0759 & 0.1103 & 0.0717 \\
\hline
\end{tabular}

As we can see from table, the GESPVE requires less time than the FCLSU, and more time than the PVAE. However, the GESPVE performs best in terms of RMSE. In conclusion, the experimental results of the real data are consistent with the ones of synthetic data. 


\section{Conclusion}

This paper extends the partially constrained abundance estimation method [10] to produce fully constrained solution. The GESPVE incorporates the subspace projection into the process of geometric abundance estimation, and uses variable endmembers to unmix the pixels. The GESPVE is in line with least square criterion and free to DR. The tests from the simulated and real data show that the GESPVE algorithm outperforms the original PVAE [10] and FSLSU [5] algorithms in terms of spectral unmixing errors. The GESPVE has a lower computational efficiency compared to the PVAE. For relatively large number of endmembers however, which are typical of most practical situations, the GESPVE requires less time than the FCLSU.

\section{Acknowledgements}

This work was supported in part by the National High Technology Research and Development Program of China (863 Program) - remote sensing ocean ecological environment monitoring system (Grant No. 2007AA092102), in part by the National Science Foundation of China (Grant 61201454), and in part by the Mational project (Grant No. 200905029-6).

\section{References}

[1] N. Keshava and J. F. Mustard, "Spectral unmîxing", JEEE Signal Processing Magazine, vol. 19, (2002), pp. 44-57.

[2] J. M. Bioucas-Dias, A. Plaza, N. Dobigeon, M. Parente, Q. Du and P. Gader, "Hyperspectral unmixing overview: Geometrical, statistical, and sparse, regression-based approaches", IEEE Journal of Selected Topics in Applied Earth Observations and Remote Sensing, yol. 5, (2012), pp. 354-379.

[3] J. Bioucas-Dias, A. Plaza, G. Camps-Valls, P. Scheunders, N. Nasrabadi, and J. Chanussot, "Hyperspectral remote sensing data analysis and future challenges,, IEEE Geoscience and Remote Sensing Magazine, vol. 1, (2013), pp. 6-36.

[4] C.-I. Chang, "Hyperspectral imaging: techniques for spectral detection and classification", Springer (2003).

[5] D. C. Heinz and C.-I. Chang, "Fully constrained least squares linear spectral mixture analysis method for material quantification in hyperspectral imagery", IEEE Transactions on Geoscience and Remote Sensing, vol. 39, (2001), pp - 529-545.

[6] L. Miao and H. Q1, "Endmember extraction from highly mixed data using minimum volume constrained nonnegative mat $1 x$ factorization", IEEE Transactions on Geoscience and Remote Sensing, vol. 45, (2007), pp. 765-777.

[7] S. Jia and Y. Qian, "Constrained nonnegative matrix factorization for hyperspectral unmixing", IEEE Transactions on Geoscience and Remote Sensing, vol. 47, (2009), pp. 161-173.

[8] J. M. Nascimento and J. M. Dias, "Does independent component analysis play a role in unmixing hyperspectral data?', IEEE Transactions on Geoscience and Remote Sensing, vol. 43, (2005), pp. 175-187.

[9] J. W. Boardman, "Geometric mixture analysis of imaging spectrometry data", Proceedings of the 1994 International Geoscience and Remote Sensing Symposium (IGARSS'94), Surface and Atmospheric Remote Sensing: Technologies, Data Analysis and Interpretation, (1994), August 8-12; Pasadena, California.

[10]X Geng, B. Zhang, X. Zhang, and L. Zheng, "An unmixing algorithm of hyperspectral imagery based on convex simplex volume in high dimensional space", Progress in Natural Science, vol. 14, (2004), pp. 810-814.

[11] P. Honeine and C. Richard, "Geometric unmixing of large hyperspectral images: A barycentric coordinate approach”, IEEE Transactions on Geoscience and Remote Sensing, vol. 50, (2012), pp. 2185-2195.

[12]P. E. Dennison and D. A. Roberts, "Endmember selection for multiple endmember spectral mixture analysis using endmember average RMSE", Remote Sensing of Environment, vol. 87, (2003), pp. 123-135.

[13] F. Maselli, "Multiclass spectral decomposition of remotely sensed scenes by selective pixel unmixing", IEEE Transactions on Geoscience and Remote Sensing, vol. 36, (1998), pp. 1809-1820.

[14] R. Clark, G. Swayze, A. Gallaher, T. King, and W. Calvin, "The US Geological Survey digital spectral library: Version 1 (0.2 to $3.0 \mu \mathrm{m})$,'Open File Report 93-592”, US Geological Survey, (1993). 
[15] J. M. Bioucas-Dias and J. M. Nascimento, "Hyperspectral subspace identification", IEEE Transactions on Geoscience and Remote Sensing, vol. 46, (2008), pp. 2435-2445.

[16] J. C. Harsanyi and C.-I. Chang, "Hyperspectral image classification and dimensionality reduction: an orthogonal subspace projection approach", IEEE Transactions on Geoscience and Remote Sensing, vol. 32, (1994), pp. 779-785.

[17] J. M. Nascimento and J. B. Dias, "Vertex component analysis: A fast algorithm to unmix hyperspectral data", IEEE Transactions on Geoscience and Remote Sensing, vol. 43, (2005), pp. 898-910.

[18] C.-I. Chang and Q. Du, "Estimation of number of spectrally distinct signal sources in hyperspectral imagery", IEEE Transactions on Geoscience and Remote Sensing, vol. 42, (2004), pp. 608-619.

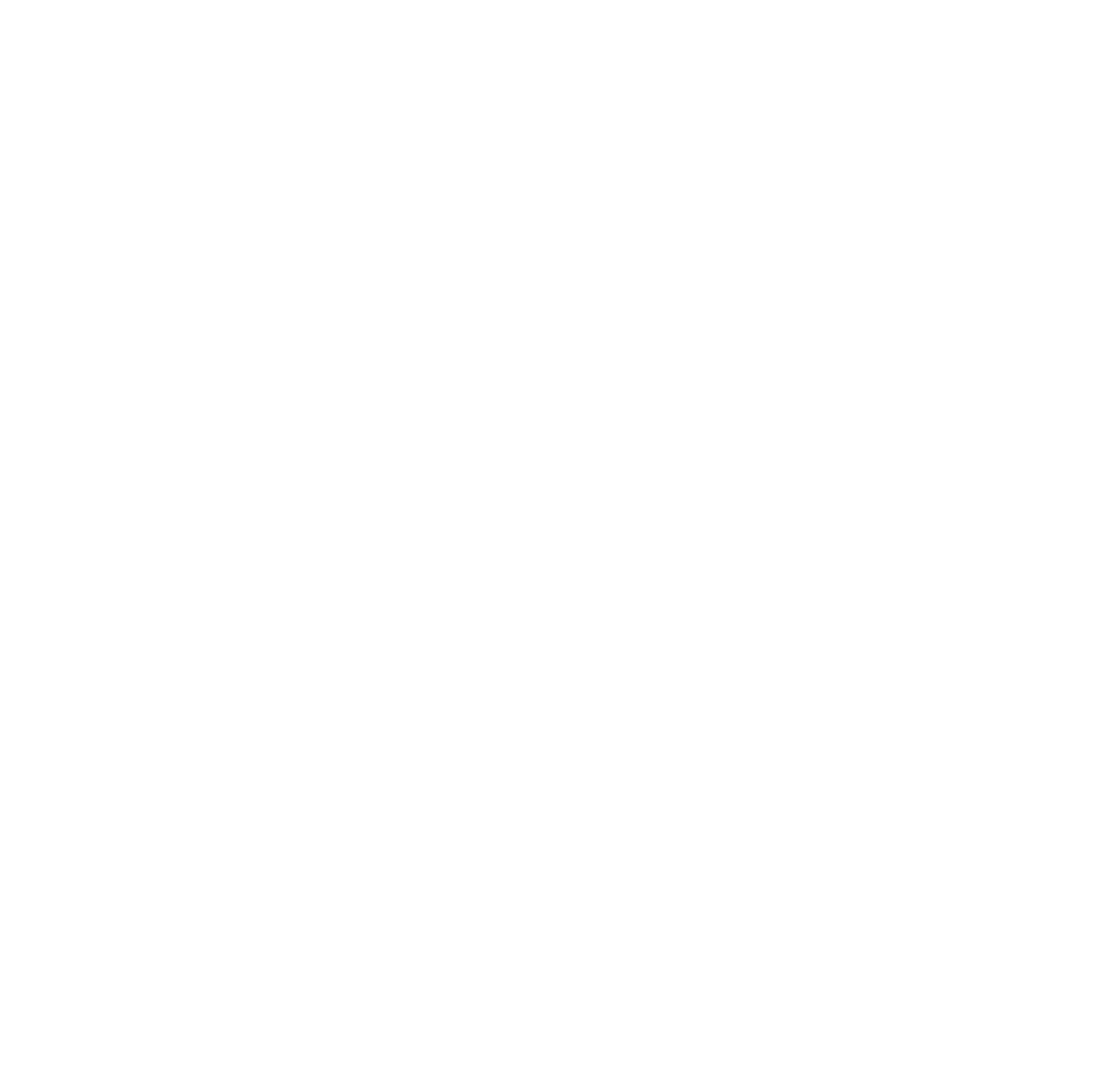

